\title{
Erratum to: An exploratory randomised double-blind and placebo-controlled phase 2 study of a combination of baclofen, naltrexone and sorbitol (PXT3003) in patients with Charcot-Marie-Tooth disease type $1 \mathrm{~A}$
}

Shahram Attarian ${ }^{1 \dagger}$, Jean-Michel Vallat ${ }^{3}$, Laurent Magy ${ }^{3}$, Benoît Funalot ${ }^{3}$, Pierre-Marie Gonnaud ${ }^{4}$, Arnaud Lacour ${ }^{5}$, Yann Péréon ${ }^{6}$, Odile Dubourg ${ }^{7}$, Jean Pouget ${ }^{1}$, Joëlle Micallef ${ }^{2}$, Jérôme Franques ${ }^{1}$, Marie-Noëlle Lefebvre ${ }^{2}$, Karima Ghorab ${ }^{3}$, Mahmoud Al-Moussawi ${ }^{4}$, Vincent Tiffreau ${ }^{5}$, Marguerite Preudhomme ${ }^{5}$, Armelle Magot ${ }^{6}$, Laurène Leclair-Visonneau ${ }^{5}$, Tanya Stojkovic ${ }^{7}$, Laura Bossi ${ }^{8}$, Philippe Lehert ${ }^{9,10}$, Walter Gilbert ${ }^{11}$, Viviane Bertrand ${ }^{12 \dagger}$, Jonas Mandel ${ }^{12}$, Aude Milet ${ }^{12}$, Rodolphe Hajj ${ }^{12}$, Lamia Boudiaf ${ }^{12}$, Catherine Scart-Grès ${ }^{12}$, Serguei Nabirotchkin ${ }^{12}$, Mickael Guedj ${ }^{12 \dagger}$, Ilya Chumakov ${ }^{12}$ and Daniel Cohen ${ }^{12^{*}}$

Unfortunately, the original version of this article [1] contained an error due to a typographical error in the computer code used. This meant the percentage improvement over baseline of the endpoints CMTNS, ONLS, 9HPT and DML was slightly increased. Therefore some of the values in Tables 3, 4, Additional file 4: Table S4 and Fig. 4 are incorrect.

However this error has no bearing on statistical outcome of the study, the conclusions or text of the manuscript. Correct versions of Tables 3, 4 and Fig. 4 can be seen below and Additional file 4 accessed using the link below.

\footnotetext{
* Correspondence: daniel.cohen@pharnext.com

${ }^{\dagger}$ Equal contributors

${ }^{12}$ Pharnext, 11, rue des Peupliers, 92130 Issy-Les-Moulineaux, Paris, France

Full list of author information is available at the end of the article
} 
Table 3 Response to PXT3003 on efficacy outcomes in treatment groups, with comparisons of active doses versus Placebo (Full Analysis Set, $n=80$ )

\begin{tabular}{|c|c|c|c|c|c|c|c|c|c|c|c|c|}
\hline & \multicolumn{4}{|c|}{ Mean \% of improvement } & \multicolumn{2}{|c|}{ PXT3003 LD versus Placebo } & \multicolumn{2}{|c|}{ PXT3003 ID versus Placebo } & \multicolumn{2}{|c|}{ PXT3003 HD versus Placebo } & \multicolumn{2}{|l|}{$\underline{\text { Dose-effect }}$} \\
\hline & Placebo & PXT3003 LD & PXT3003 ID & PXT3003 HD & Estimate & $P$-value & Estimate & $P$-value & Estimate & $P$-value & Correlation & $P$-value \\
\hline & $(n=19)$ & $(n=21)$ & $(n=21)$ & $(n=19)$ & & & & & & & & \\
\hline CMTNS & $-0.25(17.3)$ & $-3.8(20.4)$ & $-5.8(17.7)$ & $5.2(12.5)$ & $-2.6(-11.9 ; 7.6)$ & 0.67 & $-3.1(-11.0 ; 5.4)$ & 0.74 & $5.5(-3.4 ; 15.2)$ & 0.16 & 0.54 & 0.30 \\
\hline ONLS & $-11.8(33.7)$ & $-12.7(31.7)$ & $1.2(16.7)$ & $6.8(18.2)$ & $-3.9(-14.2 ; 7.6)$ & 0.72 & $6.9(-3.8 ; 18.8)$ & 0.15 & $14.4(0.55 ; 30.2)$ & $0.043^{*}$ & 0.28 & $0.006^{*}$ \\
\hline 6MWT (m) & $9.0(8.3)$ & $6.2(8.3)$ & $6.4(9.4)$ & $9.9(6.9)$ & $-2.4(-6.2 ; 1.5)$ & 0.85 & $-2.4(-6.6 ; 2.0)$ & 0.82 & $0.7(-3.2 ; 4.7)$ & 0.38 & 0.11 & 0.16 \\
\hline 9HPT (s) & $3.6(10.9)$ & $-2.5(12)$ & $4.4(9.5)$ & $6.1(10.6)$ & $-4.6(-10.3 ; 1.5)$ & 0.89 & $-0.2(-5.3 ; 5.2)$ & 0.52 & $0.3(-5.7 ; 6.6)$ & 0.47 & 0.15 & 0.092 \\
\hline Ankle Dorsiflexion (Nm) & $20.2(88.4)$ & $-3.6(43.0)$ & $81.5(369.6)$ & $20.4(64.1)$ & $-4.0(-21.7 ; 17.8)$ & 0.63 & $11.4(-15.4 ; 46.8)$ & 0.26 & $8.2(-13.8 ; 35.9)$ & 0.28 & 0.11 & 0.16 \\
\hline Grip (kg) & $9.9(24.2)$ & $1.3(15.6)$ & $4.7(12.5)$ & $11.7(18.1)$ & $-7.1(-15.6 ; 2.1)$ & 0.90 & $-3.6(-11.8 ; 5.4)$ & 0.75 & $1.6(-7.7 ; 11.9)$ & 0.39 & 0.12 & 0.15 \\
\hline CMAP (milliv) & $34.4(62.0)$ & $1.4(38.7)$ & $22.9(62.6)$ & $64.2(208.5)$ & $-25.1(-44.8 ; 1.5)$ & 0.94 & $-9.2(-27.3 ; 13.5)$ & 0.77 & $-5.1(-27.1 ; 23.6)$ & 0.63 & -0.001 & 0.50 \\
\hline $\mathrm{MCV}(\mathrm{m} / \mathrm{s})$ & $3.7(8.5)$ & $3.0(11.5)$ & $5.7(12.3)$ & $9.0(17.6)$ & $-1.0(-6.5 ; 4.9)$ & 0.61 & $0.5(-4.8 ; 6.2)$ & 0.44 & $2.8(-3.4 ; 9.4)$ & 0.23 & 0.11 & 0.18 \\
\hline DML (ms) & $-0.33(8.7)$ & $0.33(16.1)$ & $8.3(18.1)$ & $5(15.2)$ & $3.4(-4.3 ; 11.7)$ & 0.24 & $13.8(4.2 ; 24.3)$ & $0.009^{*}$ & $8.0(0.59 ; 16.0)$ & $0.038^{*}$ & 0.21 & $0.035^{*}$ \\
\hline SNAP (microV) & $12.4(121.7)$ & $11.5(88.2)$ & $23.3(128.4)$ & $5.2(69.0)$ & $-1.2(-42.9 ; 71.0)$ & 0.52 & $8.7(-31.2 ; 71.6)$ & 0.38 & $13.9(-24.1 ; 71.0)$ & 0.29 & 0.09 & 0.30 \\
\hline $\mathrm{SCV}(\mathrm{m} / \mathrm{s})$ & $3.4(11.0)$ & $5.3(11.2)$ & $29.5(63.4)$ & $30.5(10.0)$ & $1.5(-5.8 ; 9.4)$ & 0.36 & $17.5(-5.5 ; 46.2)$ & $0.11+$ & $26.6(15.5 ; 38.8)$ & $0.00037^{*}$ & 0.42 & $0.01 *$ \\
\hline
\end{tabular}

Data are mean \% (s.d.) of improvement for each treatment group. Differences between treatment groups were assessed by Analysis of Covariance (ANCOVA) on log-transformed values by adjusting for baseline values. Estimates were provided as mean percentage change over baseline $(90 \% \mathrm{Cl})$. Dose-effect was tested through Spearman's rank correlation. $P$-values are one-tailed. ${ }^{*} P<0.05$; Shading $=$ best improvement within dosages. CMTNS Charcot-Marie-Tooth Neuropathy Score, ONLS Overall Neuropathy Limitations Scale, 6MWT 6-Minute Walk Test, 9HPT 9-Hole Peg Test, CMAP Amplitudes of Compound Muscle Action Potentials, MCV Motor Conduction Velocity, DML Distal Motor Latency, SNAP Amplitudes of Sensory Nerve Action Potentials, SCV Sensitive Conduction Velocity 
Table 4 Response to PXT3003 on efficacy outcomes in HD and in PLI, with comparisons of HD versus PLI (Full Analysis Set, $n=80$ )

\begin{tabular}{|c|c|c|c|c|}
\hline & Mean $\%$ of im & & PXT3003 HD vers & \\
\hline & PLI & PXT3003 HD & Estimate & $P$-value \\
\hline & $(n=61)$ & $(n=19)$ & & \\
\hline CMTNS & $-3.4(18.4)$ & $5.2(12.5)$ & $8.0(0.4 ; 16.2)$ & $0.042^{*}$ \\
\hline ONLS & $-7.7(28.5)$ & $6.8(18.2)$ & $12.1(2.0 ; 23.2)$ & $0.024^{*}$ \\
\hline 6MWT (m) & $7.1(8.6)$ & $9.9(6.9)$ & $2.6(-0.73 ; 6.1)$ & 0.099 \\
\hline 9HPT (s) & $1.8(11.1)$ & $6.1(10.6)$ & $1.2(-3.4 ; 6.0)$ & 0.33 \\
\hline Ankle Dorsiflexion (Nm) & $33.1(223.2)$ & $20.4(64.1)$ & $5.5(-12.8 ; 27.7)$ & 0.32 \\
\hline Grip (kg) & $5.1(17.9)$ & $11.7(18.1)$ & $6.0(-1.2 ; 13.7)$ & 0.088 \\
\hline CMAP (milliv) & $19.6(56.5)$ & $64.2(208.5)$ & $6.6(-15.8 ; 35.1)$ & 0.33 \\
\hline $\mathrm{MCV}(\mathrm{m} / \mathrm{s})$ & $4.2(10.9)$ & $9.0(17.6)$ & $2.5(-2.4 ; 7.7)$ & 0.21 \\
\hline DML (ms) & $3(15.3)$ & $5(15.2)$ & $2.2(-5.1 ; 10.0)$ & 0.31 \\
\hline SNAP (microV) & $15.9(110.2)$ & $5.2(69.0)$ & $12.0(-23.9 ; 64.9)$ & 0.31 \\
\hline $\mathrm{SCV}(\mathrm{m} / \mathrm{s})$ & $12.7(38.0)$ & $30.5(10.0)$ & $20.1(2.4 ; 40.8)$ & $0.030^{*}$ \\
\hline
\end{tabular}

Data are mean \% (s.d.) of improvement for HD and for PLI after 12 months. Differences between treatment groups were assessed by Analysis of Covariance (ANCOVA) on log-transformed values by adjusting for baseline values. Estimates were provided as mean percentage change over baseline ( $90 \% \mathrm{Cl}$ ). $P$-values are one-tailed. ${ }^{*} P<0.05$; Shading = best improvement within groups. CMTNS Charcot-Marie-Tooth Neuropathy Score, ONLS Overall Neuropathy Limitations Scale, 6MWT 6-Minute Walk Test, 9HPT 9-Hole Peg Test, CMAP Amplitudes of Compound Muscle Action Potentials, MCV Motor Conduction Velocity, DML Distal Motor Latency, SNAP Amplitudes of Sensory Nerve Action Potentials, SCV Sensitive Conduction Velocity
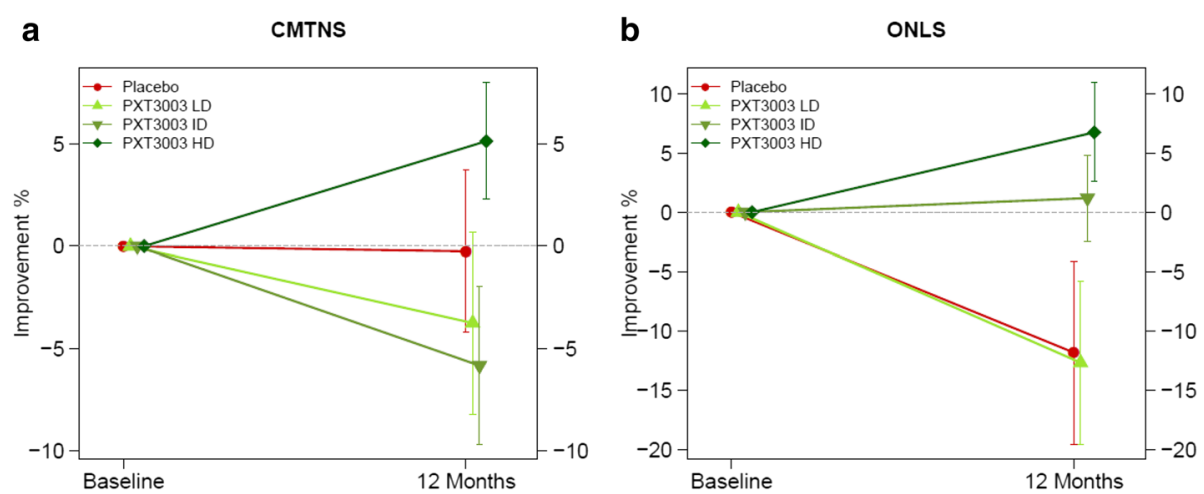

Fig. 4 Response to PXT3003 on clinical scales (Full Analysis Set, $n=80$ ). Mean \% (s.e.m.) of improvement from baseline per group at 12 months for CMTNS (a) and ONLS (b). Sample sizes: Placebo $(n=19), \operatorname{LD}(n=21), \operatorname{ID}(n=21), \mathrm{HD}(n=19)$ 


\section{Additional file}

Additional file 4: Table S4. Response to PXT3003 on efficacy outcomes (Full Analysis Set, $n=80$ ). Data are mean (s.d.) baseline and final values, and \% (s.d.) of improvement for each treatment group and PLI.

Differences between treatment groups were assessed by Analysis of Covariance (Ancova) on log-transformed values by adjusting for baseline values. Estimates were provided as mean percentage change over baseline $(90 \% \mathrm{Cl})$. Dose-effect was tested through Spearman's rank correlation. P-values are one-tailed. CMTNS = Charcot-Marie-Tooth Neuropathy Score; ONLS = Overall Neuropathy Limitations Scale; 6MWT = 6-Minute Walk Test; 9HPT = 9-Hole Peg Test; CMAP = Amplitudes of Compound Muscle Action Potentials; MCV = Motor Conduction Velocity; $\mathrm{DML}=$ Distal Motor Latency; SNAP = Amplitudes of Sensory Nerve Action Potentials; SCV = Sensitive Conduction Velocity; VAS = Visual Analog Scale; $\mathrm{CGI}=$ Clinical Global Impression. (DOC $168 \mathrm{~kb}$ )

\section{Author details}

${ }^{1}$ Centre de référence des maladies neuromusculaires et de la SLA, Pôle des neurosciences Cliniques, AP-HM et Aix Marseille Université, Marseille, France. ${ }^{2} \mathrm{CIC}$-Centre de Pharmacologie Clinique et D'Evaluations Therapeutiques, AP-HM et Aix Marseille Université, Marseille, France. ${ }^{3} \mathrm{CHU}$ de Limoges Hôpital Dupuytren, 2 Avenue Martin Luther King, 87042 Limoges, France. ${ }^{4} \mathrm{CHU}$ Lyon Sud, 165 Chemin du Grand Revoyet, 69495 Lyon, France. ${ }^{5} \mathrm{CHRU}$ de Lille - Hôpital Roger Salengro, rue Emile Laine, 59037 Lille, France. ${ }^{6} \mathrm{CHU}$ de Nantes - Hôtel Dieu, 1 place Alexis Ricordeau, 44093 Nantes, France. ${ }^{7} \mathrm{CHU}$ de Paris - Groupe Hospitalier Pitié-Salpétrière, 47-83 boulevard de l'Hôpital 75013 Paris, France. ${ }^{8}$ Admissions, 75017 Paris, France. ${ }^{9}$ Faculty of Medicine, The University of Melbourne, Grattan St, Melbourne, VIC 3010, Australia.

${ }^{10}$ Faculty of Economics, UCL Mons, Louvain, Belgium. ${ }^{11}$ Carl M. Loeb University Professor Emeritus, Harvard University, Cambridge, MA 02138, USA.

${ }^{12}$ Pharnext, 11, rue des Peupliers, 92130 Issy-Les-Moulineaux, Paris, France.

Received: 16 May 2016 Accepted: 16 May 2016

Published online: 07 July 2016

\section{References}

1. Attarian S, Vallat JM, Magy L, Funalot B, Gonnaud PM, Lacour A, Péréon Y, Dubourg O, Pouget J, Micallef J, Franques J, Lefebvre MN, Ghorab K, Al-Moussawi M, Tiffreau V, Preudhomme M, Magot A, Leclair-Visonneau L, Stojkovic T, Bossi L, Lehert P, Gilbert W, Bertrand V, Mandel J, Milet A, Hajj R, Boudiaf L, Scart-Grès C, Nabirotchkin S, Guedj M, Chumakov I, Cohen D. An exploratory randomised double-blind and placebo-controlled phase 2 study of a combination of baclofen, naltrexone and sorbitol (PXT3003) in patients with Charcot-Marie-Tooth disease type 1A. Orphanet J Rare Dis. 2014:9:199.

\section{Submit your next manuscript to BioMed Central} and we will help you at every step:

- We accept pre-submission inquiries

- Our selector tool helps you to find the most relevant journal

- We provide round the clock customer support

- Convenient online submission

- Thorough peer review

- Inclusion in PubMed and all major indexing services

- Maximum visibility for your research

Submit your manuscript at www.biomedcentral.com/submit
Biomed Central 